\title{
Symmetry indicators for topological superconductors
}

\section{Refined symmetry indicators for topological superconductors in all space groups}

Authors: S. Ono, H. C. Po, H. Watanabe

arXiv:1909.09634

\section{Symmetry-based indicators for topological Bogoliubov-de Gennes Hamiltonians}

Authors: M. Geier, P. W. Brouwer and L. Trifunovic

arXiv:1910.11271

\section{Recommended with a Commentary by Raquel Queiroz and Ady Stern, Weizmann Institute of Science}

When we study topological matter (just as in many other contexts), there is a compelling desire to obtain the maximum amount of information at the minimal amount of effort. This principle could have been coined "The principle of least action" had the term not been already used to mean something of even broader applicability. In the context of topological aspects of Bloch bands, a lot of information can be obtained when the topological invariants are known. Unfortunately, the calculation of these invariants requires much effort, involving the evaluation of the Bloch wave functions for all momenta within a Brillouin zone, followed by differentiation and integration over various combinations of these wave functions.

This commentary deals with indicators for topology, a compromise that gives less information but requires far less effort. Symmetry indicators of topology are particularly suitable for systems that possess spatial symmetries. The reduced effort they require is easy to demonstrate: rather than inferring topology from the entire set of wave functions at all momenta, the calculation of the topological indicators requires only high-symmetry momenta, those momenta that are invariant to the action of the spatial symmetry. This approach was initiated in the seminal works of $\mathrm{Fu}$ and Kane [1] in the context of inversion symmetric insulators, and of Bradlyn et. al. [2] and Po et. al. [3] who constructed generalized tools which can be applied to all space groups. These works are now used extensively to diagnose topology in real materials $[9,10,11]$.

An example to this approach is found in the Chern number, the quantized Hall conductivity, of a two dimensional system. The calculation of the Chern number requires integration over the Berry curvature at all momenta, and the Berry curvature involves scalar products of derivatives of the Bloch functions with respect to momenta. In contrast, if the system 
possesses inversion symmetry, which maps $k$ to $-k$, the total parity of the inversion symmetry eigenvalues of the wave functions at the high symmetry momenta $k_{x, y}=0, \pi$ are also the parity of the Chern number. If this parity is odd, the system is necessarily in a topological quantum Hall state, while being even leaves open the possibility of a trivial state. Inversion symmetry then allows for a symmetry indicator of topology in the form of the symmetry properties of a finite small number of Bloch wave functions.

The quantized Hall state is a relatively straightforward example to handle. For other classes the analysis is harder, and the subtlety starts already from the basic distinction between trivial and topological. A band insulator is a system of non-interacting electrons occupying an integer number of bands, such that the ground state is separated from the excited state by an energy gap. A trivial insulator is an insulator that may be deformed into an atomic insulator without the energy gap being closed throughout the process. And an atomic insulator is one in which the electrons do not hop between unit cells. In the presence of spatial symmetries, different trivial insulators may be distinguished by different sets of symmetry eigenvalues at their high symmetry momenta. The collection of symmetry eigenvalues of wavefunctions compatible with trivial atomic insulators, $\boldsymbol{a}$, can be computed comprehensively. We can define a group, which we call $\{\mathrm{AI}\}$, where these sets of symmetry eigenvalues are group elements, and where group multiplication amounts to adding or removing bands. This group is a subgroup of the group $\{\mathrm{BS}\}$, comprised of all sets symmetry eigenvalues $\boldsymbol{b}$ that are compatible with band structures. It follows that a system whose set of high-symmetry eigenvalues belong to the quotient group $\{\mathrm{SI}\}=\{\mathrm{BS}\} /\{\mathrm{AI}\}$ is necessarily topological, while a system whose set of eigenvalues are part of $\{\mathrm{AI}\}$ may be either trivial or topological. The sets that belong to $\{\mathrm{SI}\}$ are then the symmetry indicators.

The generalization of this method to superconductors, which is the subject of the two papers we recommend here, poses a hard challenge. Although symmetry criteria for topological superconductivity were proposed before $[4,5,6]$, a complete study of all space groups is only now being carried out. The Kitaev chain [7] is the canonical example for a topological superconductor, and is suitable for exemplifying the challenge involved. It is composed of a one dimensional lattice with a single electronic state per site, in which the pairing interaction is between nearest-neighboring sites. It enjoys a symmetry to inversion, both with the inversion center being on a lattice site and with the inversion center being mid-way between sites. When the eigenvalues of inversion at the high symmetry momenta $k=0, \pi$ are calculated, their values depend both on the position of the center of inversion and on whether the chain is in its trivial or topological state. Specifically, the inversion eigenvalues obtained when the center of inversion is on a lattice site and the chain is in a trivial phase are identical to those that are obtained when the center of inversion is mid-way between sites and the chain is in a topological state. Given that the system is symmetric to both types of inversion, one wonders whether this is an indication that symmetry indicators do not indicate anything for topological superconductivity. Put differently, does the subgroup $\{\mathrm{AI}\}$ consume the entire group of possible band structures $\{\mathrm{BS}\}$, and leaves no indicators that identify states as topological?

Fortunately, the two articles we recommend in this commentary give a negative answer to this question. Both Ono et. al. and Geier et. al. tell us that what we lack in directly applying the tool of symmetry indicators to superconductors is a frame of reference. In the example we outlined in the previous paragraph we witnessed the difficulty of using symmetry 
eigenvalues to distinguish between, on one hand, driving a Kitaev chain through a transition from a trivial state to a topological state, and on the other hand, shifting it by half a unit cell. This difficulty is remedied if we add to the Kitaev chain, in parallel, a band of fully localized electrons. The inversion eigenvalues of this band are flipped if the system is shifted by half a unit cell, while they do not change if the Kitaev chain is driven through the phase transition. Thus, in the combined system inversion symmetry eigenvalues are indicators for topology. The fully localized band we added here to the Kitaev chain is an example to a reference frame, a state we unambiguously define as trivial, and with respect to we measure the triviality of other gapped states.

Diving minimally into details, let us recall that under a spatial symmetry $g$ in the space group, a Bogolubov-de Gennes (BdG) Hamiltonian is transformed in orbital space with a projective representation $U_{k}(g)=\operatorname{diag}\left(u_{k}(g),-\xi u_{-k}^{*}(g)\right)$ and its momentum is transformed from $k$ to $g k$. The normal and superconducting terms transform as $h_{k}=u_{k}(g) h_{g k} u_{k}(g)^{\dagger}$ and $\Delta_{k}=\xi^{*} u_{k}(g) \Delta_{g k} u_{k}(g)^{T}$, respectively. Furthermore, the Hamiltonian satisfies a particle-hole conjugation, here represented by a bar. Explicitly in the example of the Kitaev chain with inversion symmetry, the Hamiltonian is written as $H_{k}=(m-\cos k) \tau_{z}+\Delta \sin k \tau_{x}$, where electrons in an inversion-symmetric state are paired by an order parameter that is odd under inversion, such that $\xi=-1$. In this case $U_{k}(I)=\tau_{z}$, and particle-hole antiunitary transformation is $K \tau_{x}$ (with $K$ being complex conjugation).

Ono et. al. provide us with a simple formula to diagnose whether or not a particular $\boldsymbol{b}$ may be adiabatically connected to a reference trivial insulator, described by $\boldsymbol{b}^{\mathrm{vac}}$, either by a variation of the Hamiltonian's parameters or by adding in trivial degrees of freedom contained in the $\{\mathrm{AI}\}$ set. This relation states,

$$
\boldsymbol{b}-\boldsymbol{b}^{\mathrm{vac}}=\boldsymbol{a}-\overline{\boldsymbol{a}} .
$$

The topological content captured by this relation goes beyond the simple computation of the spatial symmetry eigenvalues and matching them with those of atomic insulators. It allows us to identify elements of $\{B S\}$ which belong to the subgroup $\{\mathrm{AI}\}$ : We label any set in $\{\mathrm{BS}\}$ in terms of the number of states below the chemical potential with positive and negative eigenvalues of inversion at the high symmetry points $k=0, \pi$, which we write as $\boldsymbol{b}=\left\{n_{+}^{0}, n_{-}^{0}, n_{+}^{\pi}, n_{-}^{\pi}\right\}$. For gapped systems $n_{+}^{0}+n_{-}^{0}=n_{+}^{\pi}+n_{-}^{\pi}$. As before, the position of the lattice with respect to the center of inversion affects the symmetry representations. In onedimensional charge-conserving insulators with no symmetry but inversion, the right hand side of (1) is only $\boldsymbol{a}$, and as a consequence all combinations $\boldsymbol{b}$ represent atomic insulators, with exponentially localized Wannier orbitals, leading to $\{\mathrm{AI}\}=\{\mathrm{BS}\}$. For superconductors, however, the situation is different, since the right hand side of Eq.(1) imposes a more stringent condition on $\boldsymbol{b}$. Choosing the reference atomic insulator to be $\boldsymbol{b}^{\mathrm{vac}}=\{1,0,1,0\}$, we may compute the space comprised by the BdG Hamiltonians of atomic insulators, by using the elements $\boldsymbol{a}-\overline{\boldsymbol{a}}$. A lattice of electrons with the inversion centers at a lattice site corresponds to $(\boldsymbol{a}-\overline{\boldsymbol{a}})_{0}=\{1,-1,1,-1\}$, while an inversion center mid-way between lattice sites corresponds to $(\boldsymbol{a}-\overline{\boldsymbol{a}})_{1 / 2}=\{-1,1,1,-1\}$. The group $\{\mathrm{AI}\}$ comprises of all $\boldsymbol{b}$ 's that may be built of these ingredients. Now, looking at the Kitaev chain (with $m>0$ for convenience), we find that in its topological phase $(m<1)$ its symmetry eigenvalues are $\boldsymbol{b}^{\text {top }}=\{1,0,0,1\}$, while in its trivial phase $(m>1)$ they are $\boldsymbol{b}^{\text {tr }}=\{1,0,1,0\}$. While $\boldsymbol{b}^{\text {tr }}$ belongs to $\{\mathrm{AI}\}$, the topological 
$\boldsymbol{b}^{\text {top }}$ does not, since it cannot be obtained from Eq.(1) with an $\boldsymbol{a}-\overline{\boldsymbol{a}}$ that corresponds to a gapped system.

Finally, both papers leave open the possibility of using their analysis to study possible fragile topological superconductors, akin to the fragile topological insulators found in the context of charge-conserving systems [8]. An intriguing question indeed!

\section{References}

[1] Fu, L., Kane, C. L. Topological insulators with inversion symmetry. Phys. Rev. B 76, 045302 (2007).

[2] Bradlyn, B. et al. Topological quantum chemistry. Nature 547, 298305 (2017).

[3] Po, H. C., Vishwanath, A., Watanabe, H. Symmetry-based indicators of band topology in the 230 space groups. Nat. Commun. 8, 50 (2017).

[4] Fu, L., Berg, E. Odd-parity topological superconductors: Theory and application to $\mathrm{Cu}_{\mathrm{x}} \mathrm{Bi}_{2} \mathrm{Se}_{3}$. Phys. Rev. Lett. 105, 14 (2010).

[5] Sato, M. Topological odd-parity superconductors. Phys. Rev. B 81, 220504 (2010).

[6] Skurativska, A., Neupert, T. Fischer, M. H. Atomic limit and inversion-symmetry indicators for topological superconductors. Phys. Rev. Res. 2, 13064 (2020).

[7] Kitaev, A. Y. Unpaired Majorana fermions in quantum wires. Physics-Uspekhi 44, 131136 (2001).

[8] Po, H. C., Watanabe, H., Vishwanath, A. Fragile Topology and Wannier Obstructions. Phys. Rev. Lett. 121, 126402 (2018).

[9] Tang, F., Po, H. C., Vishwanath, A., Wan, X. Comprehensive search for topological materials using symmetry indicators. Nature 566, 486489 (2019).

[10] Zhang, T. et al. Catalogue of topological electronic materials. Nature 566, 475479 (2019).

[11] Vergniory, M. G. et al. A complete catalogue of high-quality topological materials. Nature 566, 480485 (2019). 